\section{Triggering risk factors for ischemic stroke}

Koton et al. studied the association between exposure to seven potential triggers and ischemic stroke onset. Thirtyeight percent of patients reported exposure to at least one potential trigger during the 2-hour hazard period. Triggers with elevated odds ratios: negative emotions; anger; and sudden changes in body posture in response to a startling event.

see page 2006

\section{Medical treatment following carotid endarterectomy}

In the years after patients have carotid endarterectomy (CE), they remain at risk for ischemic vascular events. Betancourt et al. assessed the use of medications proven to reduce vascular risk in the 1-year period following CE. Use of statins, angiotensin-converting enzyme inhibitors, and antiplatelet agents was suboptimal.

see page 2011

\section{Is intracranial arterial dolichoectasia a systemic disease?}

In this MRI case control study of stroke patients, Pico et al. showed that intracranial arterial dolichoectasia was associated with enlarged descending thoracic aorta by TEE with a dose response relationship. The underlying process causing intracranial arterial dolichoectasia may affect the descending thoracic aorta.

see page 2016

\section{IVIG for acute multiple sclerosis (MS) relapses}

Soelberg Sorensen et al. treated 76 MS patients who had suffered an acute relapse with either IV immunoglobulins (IVIG) $1 \mathrm{~g} / \mathrm{kg}$ or placebo 24 hours before treatment with IV methylprednisolone. Both groups improved, but without any difference between IVIG and placebo.

see page 2028

\section{Long-term exercise and multiple sclerosis (MS)}

Romberg et al. studied the effects of a 6-month progressive exercise program on functional ability of patients with MS. Regular exercise was associated with clinically meaningful changes in walking speed and had no detrimental effect on MS activity.

\section{Clustering of sporadic CJD in the UK}

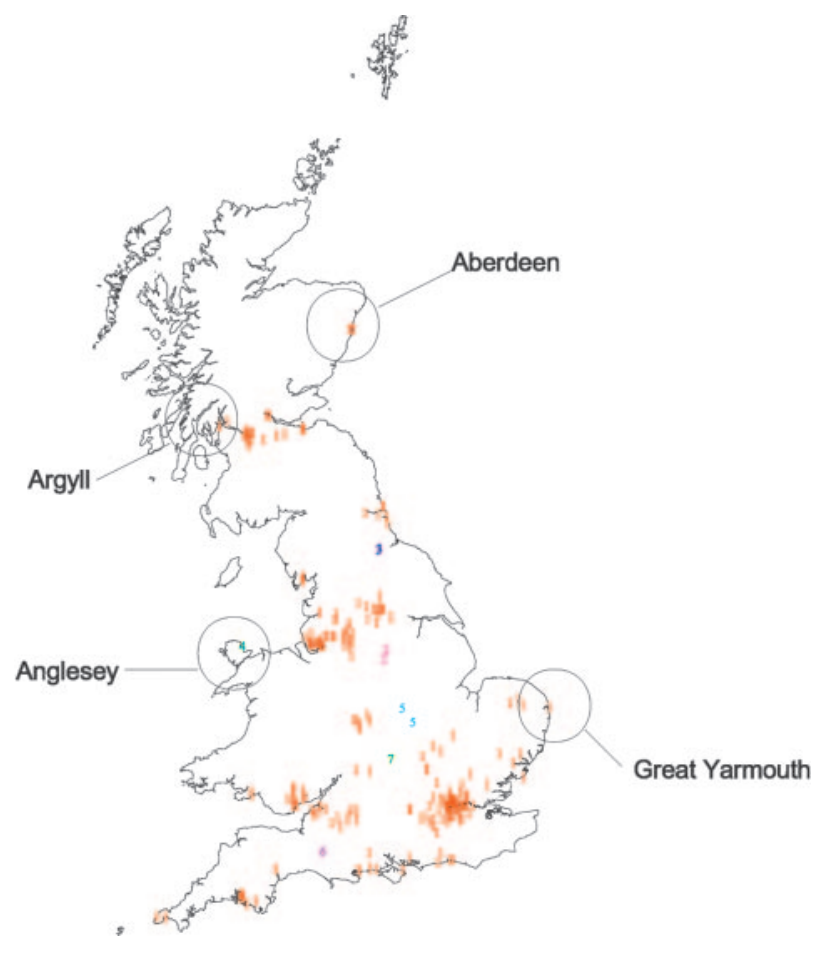

Linsell et al. investigated lifetime residential histories of sporadic Creutzfeldt-Jakob disease (CJD) cases. Cases lived closer to each other than would be expected in the absence of a disease clustering mechanism. This finding suggests that some sporadic CJD cases result from exposure to an external factor.

see page 2077

\section{Markedly elevated GAD antibodies in SPS}

Murinson et al. studied glutamic acid decarboxylase (GAD) antibodies in 576 patients with suspected stiffperson syndrome (SPS). Patients with SPS showed marked elevations of GAD antibodies by radioimmunoassay (RIA), the levels of which did not vary with patient age or symptom duration.

see page 2146

The accompanying editorial by Chang and Lang notes that the Murinson et al. data do not imply a pathogenic role for GADAbs, since they found no correlation between age or duration of illness and GADAbs, and no change over the course of the disease in individuals. Thus there is no value in monitoring the antibody titer during the course of disease. The exact role of GADAbs in the pathogenesis of SPS remains elusive, but Murinson et al. have established the reliability of RIA in measuring these antibodies. 


\section{Pregabalin for diabetic neuropathy}

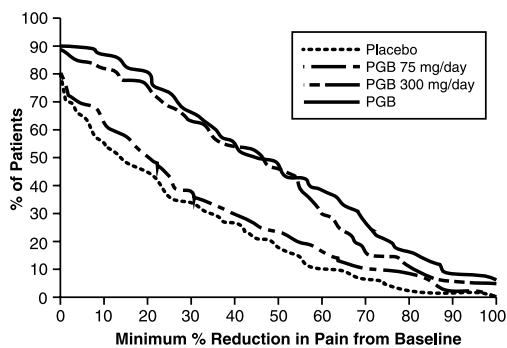

Lesser et al. evaluated pregabalin as treatment for painful diabetic peripheral neuropathy in a randomized controlled trial in 338 patients. Dosages of 300 and 600 $\mathrm{mg} /$ day were well tolerated, produced significant, early, and sustained improvements in pain and sleep interference, and were significantly superior to placebo on patient and clinical global impressions of change.

see page 2104

\section{- Asomatognosia: Possible cause of falls and injuries in epilepsy}

Epileptic falls are often thought to be due to motor convulsions or weakness. This report by So and Schaüble describes the use of multiple modalities of electrophysiologic tests in revealing asomatognosia during seizures as a cause of repeated falls and serious injuries.

see page 2153

\section{Unexpected suicides in successful DBS for movement disorders}

Burkhard et al. report six cases of suicide among 140 patients successfully treated with deep brain stimulation (DBS) for movement disorders. Five had a history of depression. Suicide occurred despite the success of DBS.

see page 2170

\section{Spasticity and intensive training regimes in hemiplegia treatment}

In contrast to the common assumption that movement practice can enhance existing spasticity, Sterr and Freivogel found that intensive affected arm training in chronic hemiplegia had no adverse effects on muscle tone.

see page 2176
Evidence that space is shifted, not rotated in spatial neglect

Richard et al. investigated how right-brain-damaged patients represented the straight-ahead direction using the manipulation of a horizontal rod. Results suggested a preferential rightward translation without rotation of space representation.

see page 2136

\section{Right parietal cortex and numerical space representation}

Oliveri et al. show that numerical distances look bigger in the left side of space. The right parietal cortex is associated with the overestimation of left-side mental spatial representation of quantity.

see page 2139

The editorial accompanying these two papers by Heilman, Jeong, and Finney notes that the experiment by Richard et al., elegantly demonstrates that the primary alteration of the spatial representation of a person's mid-sagittal plane in leftsided neglect is a shift to the right, though it is still possible that some subsets of neglect, a heterogeneous syndrome, may involve an element of rotation as well. The work of Oliveri et al., demonstrates that a left-sided bias is not limited to external stimuli but also occurs in imagery, and this bias is being induced primarily by the right parietal lobe.

see page 1994

\section{Predicting early progression in MCA infarcts}

Maramattom et al. studied 24 patients with complete middle cerebral artery infarction (coMCAI). Female gender or additional vascular involvement on CT were risk factors for further deterioration. These features may identify patients at high risk of deterioration after coMCAI.

see page 2142

The accompanying editorial by Diringer and Kaufmann notes that although hemicraniectomy was abandoned decades ago, improvement in surgical techniques have led to growing use of hemicraniectomy in patients with ischemic stroke, head injury, and intracranial hemorrhage. It is essential to be able to accurately identify patients likely to deteriorate and fail to respond to a trial of medical management. Maramattom et al. add a tool in the identification of patients likely to deteriorate from edema following large hemispheric stroke. Two factors predicted further deterioration in their series: female gender (72 vs 20\%) and additional vascular territory beyond coMCAl (72 vs 0\%). However, the study is uncontrolled, and does not indicate what constitutes a reasonable trial of medical management. The potential of hemicraniectomy and durotomy vs medical management alone to improve survival must be tested with a well-designed randomized clinical trial.

see page 1997 


\section{Neurology}

\section{December 14 Highlights}

Neurology 2004;63;1992-1993

DOI 10.1212/WNL.63.11.1992

This information is current as of December 13, 2004

\section{Updated Information \&}

Services

Permissions \& Licensing

Reprints including high resolution figures, can be found at: http://n.neurology.org/content/63/11/1992.full

Information about reproducing this article in parts (figures,tables) or in its entirety can be found online at:

http://www.neurology.org/about/about_the_journal\#permissions

Information about ordering reprints can be found online:

http://n.neurology.org/subscribers/advertise

Neurology ${ }^{\circledR}$ is the official journal of the American Academy of Neurology. Published continuously since 1951, it is now a weekly with 48 issues per year. Copyright. All rights reserved. Print ISSN: 0028-3878. Online ISSN: 1526-632X.

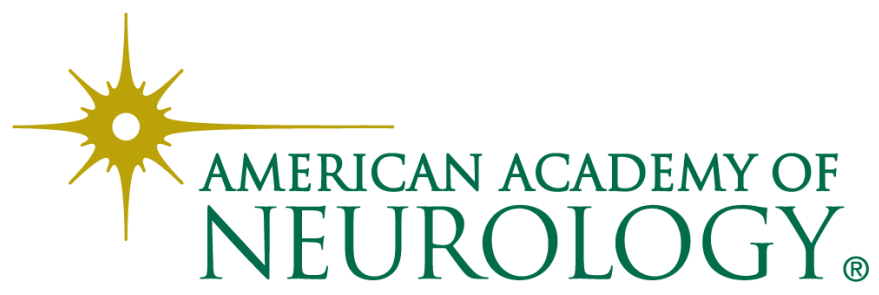

